See discussions, stats, and author profiles for this publication at: https://www.researchgate.net/publication/258086218

\title{
Enhanced Cooperative Interactions at the Nanoscale in Spin-Crossover Materials with a First-Order Phase Transition
}

Article in Physical Review Letters · June 2013

DOI: 10.1103/PhysRevLett.110.235701

CITATIONS

67

10 authors, including:

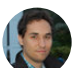

Gautier Félix

French National Centre for Scientific Research

27 PUBLICATIONS 454 CITATIONS

SEE PROFILE

Marine Perrier

Université de Montpellier

10 PUBLICATIONS 166 CITATIONS

SEE PROFILE
READS

121

William Nicolazzi

Laboratoire de Chimie de Coordination.

58 PUBLICATIONS 2,354 CITATIONS

SEE PROFILE

Guillaume Maurin

Université de Montpellier

373 PUBLICATIONS 19,988 CITATIONS

SEE PROFILE

Some of the authors of this publication are also working on these related projects:

MOFs as nanoDampers or nanoSprings: an exploration of the thermodynamic and mechanicallproperties of Metal Organic Frameworks View project

ZnO films and nanoparticles View project 


\title{
Enhanced Cooperative Interactions at the Nanoscale in Spin-Crossover Materials with a First-Order Phase Transition
}

\author{
Gautier Félix, ${ }^{1}$ William Nicolazzi, ${ }^{1}$ Lionel Salmon, ${ }^{1}$ Gábor Molnár, ${ }^{1}$ Marine Perrier, ${ }^{2}$ Guillaume Maurin, ${ }^{2}$ \\ Joulia Larionova, ${ }^{2}$ Jérôme Long, ${ }^{2}$ Yannick Guari, ${ }^{2}$ and Azzedine Bousseksou ${ }^{1, *}$ \\ ${ }^{1}$ LCC, CNRS and Université de Toulouse (UPS, INP), 205 route de Narbonne, F-31077 Toulouse, France \\ ${ }^{2}$ Institut Charles Gerhardt Montpellier, UMR 5253 CNRS-UM2-ENSCM-UM1, Chimie Moléculaire et Organisation du Solide, \\ Université Montpellier II, 34095 Montpellier, France \\ (Received 18 February 2013; published 4 June 2013)
}

\begin{abstract}
We analyzed the size effect on a first-order spin transition governed by elastic interactions. This study was performed in the framework of a nonextensive thermodynamic core-shell model. When decreasing the particle size, differences in surface energies between the two phases lead to the shrinking of the thermal hysteresis width, the lowering of the transition temperature, and the increase of residual fractions at low temperature, in good agreement with recent experimental observations on spin transition nanomaterials. On the other hand, a modification of the particle-matrix interface may allow for the existence of the hysteresis loop even at very low sizes. In addition, an unexpected reopening of the hysteresis, when the size decreases, is also possible due to the hardening of the nanoparticles at very small sizes, which we deduced from the size dependence of the Debye temperature of a series of coordination nanoparticles.
\end{abstract}

DOI: 10.1103/PhysRevLett.110.235701

PACS numbers: $64.70 . \mathrm{Nd}, 62.20 . \mathrm{de}, 75.30 . \mathrm{Wx}$

The fundamental understanding and the control of physical and chemical properties of phase transition materials at the nanometer scale are the object of significant research activity [1]. Decreasing the size of these materials to the nanometer scale gives rise to new phenomena with respect to phase stability. These phenomena include a variety of confinement effects as well as the inexorably increasing role of the interface energy in the thermodynamical properties of the material [2-5]. In addition, the phase transformation kinetics can be also strongly altered at reduced sizes [6]. The modulation of the phase diagram when approaching the nanometer scale may be so important that new phases with new properties appear which cannot be observed in the bulk materials $[4,5]$. Indeed a number of experimental and theoretical studies confirmed these ideas, a particularly well documented example being the melting transition of nanocrystals $[2,3]$.

In this Letter, we focus on an emerging class of nanomaterials displaying the molecular spin-crossover (SCO) phenomenon. These transition metal complexes exhibit bistability between the so-called low spin (LS) and high spin (HS) electronic configurations, which is accompanied by a spectacular change of the material properties [7]. The molecular spin state change in the bulk material gives rise to elastic interactions between the molecules due to the strong electron-lattice coupling, leading to the emergence of various cooperative phenomena, such as first-order phase transitions. We focus here on these cooperative effects, because they are considered as the key properties of these smart molecular systems for future applications in memory and highly efficient switching devices. Indeed, the recent synthesis and characterization of these coordination compounds as nanoparticles, nanowires, thin films, and nanoscale assemblies have brought new fundamental questions about the control and the preservation of the cooperativity at the nanometric scale [8,9]. Recent theoretical investigations using the well-known Ising-like model predicted a decrease of the spin transition temperature, an increase of the residual HS fraction at low temperatures, and the narrowing of the hysteresis width with decreasing particle size, leading to the ineluctable loss of the hysteresis [10-13]. On the other hand, the available rare experimental results remain somewhat discordant. For example, the size-dependent thermal hysteresis curves reported in Refs. [14-17] correspond well to these theoretical predictions. In several other cases, however, a somewhat unexpected conservation of the hysteresis loop has been detected down to particle sizes as small as 3-4 nm [18-23]. Of course, these differences might just be related to the different chemical nature of the particles. In addition, the mechanical properties of the particles may be also size dependent. Another potentially important parameter is the nature of the matrix in which the particle is embedded. This has been clearly demonstrated by Raza et al. [22], who synthesized core-shell particles with the same $10 \mathrm{~nm} \mathrm{Fe}$ (pyrazine) $\left[\mathrm{Pt}(\mathrm{CN})_{4}\right]$ core and reported a strong variation of the hysteresis width as a function of the chemical nature and the thickness of the shell layer. The authors proposed that interactions between the nanoparticles may arise and can be modulated by their environment. Of course, the mechanical properties of the matrix as well as the physicochemical properties of the particlematrix interface may also have a strong influence on the observed properties [20].

In order to better understand the size dependence of the spin transition, we present here a combined experimental 
and theoretical study. We developed a thermodynamical core-shell model in which surface energy as well as elasticity are taken into account. The size effect on the elastic properties has been analyzed through the experimental determination of the Debye temperature $\left(\Theta_{\mathrm{D}}\right)$ by Mössbauer spectroscopy in three Prussian blue analogue model compounds with different nanoparticle sizes.

In the following, we consider a spherical nanoparticle with a radius $R$ constituted of a bulklike core, which is connected to a surface $S$, the so-called core-shell approach (Fig. 1, inset). This type of model is often used to account - in a simple manner-for the fact that the chemical and physical properties of nanomaterials are not homogeneous $[2,11]$. The total Gibbs free energy per mole of a nanoparticle in contact with a thermal bath at the temperature $T$ can be expressed as

$$
\begin{aligned}
G= & n_{\mathrm{HS}} G_{\mathrm{HS}}+\left(1-n_{\mathrm{HS}}\right) G_{\mathrm{LS}}-T\left(S_{\mathrm{mix}}^{b}+S_{\mathrm{mix}}^{s}\right) \\
& +S\left[\sigma_{\mathrm{HS}} n_{\mathrm{HS}}^{s}+\sigma_{\mathrm{LS}}\left(1-n_{\mathrm{HS}}^{s}\right)\right]+\Gamma n_{\mathrm{HS}}\left(1-n_{\mathrm{HS}}\right),
\end{aligned}
$$

where $n_{\mathrm{HS}}=c^{b} n_{\mathrm{HS}}^{b}+c^{s} n_{\mathrm{HS}}^{s}$ stands for the total mean number of molecules in the HS state, the so-called HS fraction, while $G_{\mathrm{HS}}$ and $G_{\mathrm{LS}}$ correspond to the Gibbs energy of the HS and LS phases, respectively. It is important to note that the introduction of surfaces leads to the loss of the extensive character of $G$ and its derivatives. In the approximation of a spherical nanoparticle where $V_{0}$ and $S_{0}$ are the unit-cell volume and surface, respectively, the $c^{s}$ and $c^{b}$ coefficients are, respectively, $c^{s}=\left(3 V_{0}\right) /\left(R S_{0}\right)$ and $c^{b}=1-c^{s}$, with $c^{s}, c^{b} \in[0,1] . S_{\text {mix }}^{b}$ and $S_{\text {mix }}^{s}$ are the mixing entropies of the bulk and the surface, respectively. The next term in Eq. (1) gives the energy cost for the creation of an interface with a surface HS fraction $n_{\mathrm{HS}}^{s}$. The quantity $\sigma_{i}(i=\mathrm{HS}, \mathrm{LS})$ is the surface (interface) energy, which can include surface stress, due to the curvature of the interface separating the nanoparticle and the

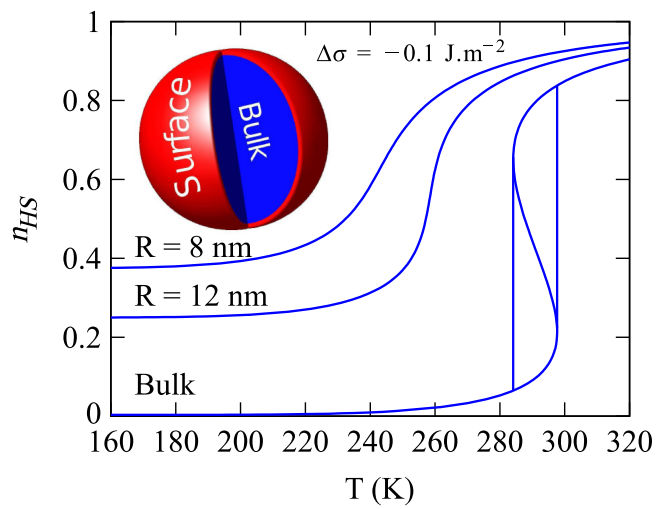

FIG. 1 (color online). Thermal evolution of the total HS fraction for different nanoparticle sizes calculated with the thermodynamic model. Parameters used (see also Supplemental Material [28]): $\Delta H=18000 \mathrm{~J} \cdot \mathrm{mol}^{-1}, \Delta S=61 \mathrm{~J} \cdot \mathrm{K}^{-1} \cdot \mathrm{mol}^{-1}$, $\Delta \sigma=-0.1 \mathrm{~J} \cdot \mathrm{m}^{-2}, \Gamma_{\mathrm{HS}}=5143 \mathrm{~J} \cdot \mathrm{mol}^{-1}, \Gamma_{\mathrm{LS}}=6857 \mathrm{~J} \cdot \mathrm{mol}^{-1}$, and $\Gamma=n_{\mathrm{HS}} \Gamma_{\mathrm{HS}}+\left(1-n_{\mathrm{HS}}\right) \Gamma_{\mathrm{LS}}$. surrounding medium [24]. The last term in Eq. (1) corresponds to the intermolecular interactions, whose strength is effectively governed by the phenomenological $\Gamma$ parameter [25]. This thermodynamical approach can be directly related to elastic mesoscopic models in the framework of continuum mechanics. In particular, it has been shown that $\Gamma$ has a linear dependence with the elastic bulk modulus $K$ in the mean field (MF) approach: $\Gamma \propto K$ [26]. In the effective medium approximation, the elastic bulk modulus is assumed to be uniform and homogeneous in the nanoparticle: $K=n_{\mathrm{HS}} K_{\mathrm{HS}}+\left(1-n_{\mathrm{HS}}\right) K_{\mathrm{LS}}$, where $K_{\mathrm{HS}}$ and $K_{\mathrm{LS}}$ stand for the bulk modulus of the HS and LS phases, respectively. This excludes clustering process, in agreement with the intrinsic MF assumptions dictated by our thermodynamical model. The bulk modulus is accessible either from high pressure x-ray diffraction or, as in the present case, can be derived from the Debye temperature $\Theta_{D}$ of the particles [27]. The stationary solutions are given by the two relations $\partial G / \partial n_{\mathrm{HS}}^{b}=0$ and $\partial G / \partial n_{\mathrm{HS}}^{s}=0$, and a unique couple $\left(n_{\mathrm{HS}}^{s}, n_{\mathrm{HS}}^{b}\right)$ exists for a given temperature $T$ (for more details of the model see the Supplemental Material [28]). The thermal evolution of the total HS fraction $n_{\mathrm{HS}}$ is depicted in Fig. 1 for different nanoparticle sizes. A decrease of the transition temperature $T_{1 / 2}$ as well as an increase of the residual HS fraction at low temperature is observed by lowering the size of the system in agreement with experimental observations reported in Refs. [14,15]. These two effects arise due to the presence of the surfaces, which can be seen as local modifications of the ligand field and/or the surface stress, attributed to chemical bond differences at the surface, a lowering of coordination number, and possible interactions with the external environment. The magnitude of these size effects is directly related to the difference between the HS and LS surface energies $\Delta \sigma=\sigma_{\mathrm{HS}}-\sigma_{\mathrm{LS}}$ [Fig. 2(a)]. If $\Delta \sigma<0$ (respectively, $\Delta \sigma>0$ ), the HS state (respectively, LS state) is favored at the surface and the transition temperature shifts to lower (respectively, higher) temperatures.
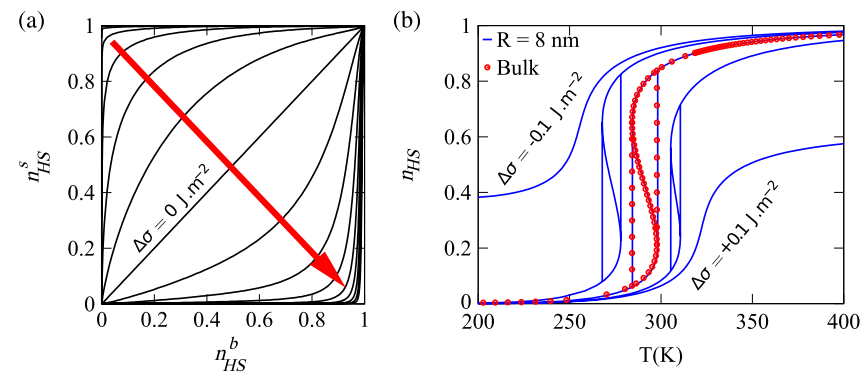

FIG. 2 (color online). (a) A couple of solutions between $n_{\mathrm{HS}}^{s}$ and $n_{\mathrm{HS}}^{b}$ for different values of $\Delta \sigma$ (varying from -0.04 to +0.04 along the arrow). (b) The symbols display the spin transition for the bulk material. The lines show the transition for an $8 \mathrm{~nm}$ nanoparticle with different surface tensions, respectively, from the left to the right: $\Delta \sigma=-0.1 \mathrm{~J} \cdot \mathrm{m}^{-2},-0.005 \mathrm{~J} \cdot \mathrm{m}^{-2}$, $0 \mathrm{~J} \cdot \mathrm{m}^{-2},+0.005 \mathrm{~J} \cdot \mathrm{m}^{-2}$, and $+0.1 \mathrm{~J} \cdot \mathrm{m}^{-2}$. 
Therefore, the surface molecular state can be partially "fixed" either in the LS or in the HS states. Actually, the surface energy does not fix the spin state of the molecules at the surface but instead changes locally the transition temperature. Therefore, the occurrence of multistep transition cannot be excluded, attributed to the transition of the core and the surface(s) of the nanoparticle. On the other hand, surface energy depends also on the bulk modulus of the nano-objects [24]: $\Delta \sigma=f(\Delta K)$, where $\Delta K=K_{\mathrm{HS}}-$ $K_{\mathrm{LS}}$. It is well known that the LS phase is more rigid than the HS phase, leading most of the time to a negative value of $\Delta \sigma$. It can be interpreted as a negative pressure which is only active on the surface [12], resulting for SCO nanoparticles in a shift of $T_{1 / 2}$ to lower temperatures and in an increase of the residual HS fraction. In the limit $\Delta \sigma \rightarrow-\infty$, the surface is totally fixed in the HS state, i.e., $n_{\mathrm{HS}}^{s} \rightarrow 1$ [see Fig. 2(a)], and an analytical expression for the equilibrium temperature $T_{1 / 2}(R)$ of nanoparticles with size $R$ can be established, assuming $\Delta K / K \ll 1$ (see demonstration in the Supplemental Material [28]):

$$
T_{1 / 2}(R)=T_{1 / 2}(\infty)-\frac{1}{R} \frac{3 V_{0}}{S_{0} \Delta S} \Gamma,
$$

where $T_{1 / 2}(\infty)$ corresponds to the transition temperature in the bulk material and $\Delta S$ stands for the entropy difference between the two states. This limit situation is equivalent to the recent numerical investigation of size effects in SCO nanoparticles using the Ising-like model with fixed boundary conditions, which constitutes a particular case of our present approach [11]. The $1 / R$ dependence of the transition temperature with the radius of the nanoparticle is a well-known result, which can be derived from the LaplaceYoung equation and which has already been observed in other finite size first-order phase transitions such as the melting process $[2,3]$.

It is important to note that a complete spin transition may be obtained even for very small particles by tuning the physical (additive stress) or chemical (modification of the ligand) properties at the surfaces. For example, the diminution of $|\Delta \sigma|$, as shown in Fig. 2(b), allows one to recover the bulk transition for an $8 \mathrm{~nm}$ particle. This effect have been already observed experimentally by modifying the surface of SCO nanoparticles [22]. In other words, a careful engineering of the particle-matrix interface may allow one to tune the spin-crossover properties of the particles and preserve the bistability even at very small sizes. Figure 2(b) reveals also the possibility to observe an increase of the transition temperature and an increase of the residual LS fraction with decreasing particle size for the case of $\Delta \sigma>0$. If now we consider a nonspherical nanoparticle, then different surfaces may also have different interfacial energies. In this case, both HS and LS residual fractions could be observed in the same nanoparticle, such as reported in Refs. [14,18].
In the previous simulations, the decrease of the hysteresis width, i.e., the loss of cooperativity, was obtained for a fixed value of the parameter $\Gamma$ (see Fig. 1). However, the elastic properties of a nanoparticle can be very different from the elasticity of the bulk material. The tendency of the variation of elastic properties with the size of the particles depends a lot on the material. For example, in the case of noble metal nanoparticles, a higher stiffness was reported when compared to the bulk material [29]. In general, the elastic modulus of ultrasmall $(<5-10 \mathrm{~nm})$ nanoparticles is enhanced [4,29], yet a diminution is also possible [5]. Therefore, a more precise analysis of the size-dependent mechanical properties of coordination nanoparticles becomes essential. To this aim, we have studied a series of nanoparticles (in polymer matrix) of three model compounds $\left(\mathrm{Fe}_{4}\left[\mathrm{Fe}(\mathrm{CN})_{6}\right], \mathrm{Ni}_{3}\left[\mathrm{Fe}(\mathrm{CN})_{6}\right]_{2}\right.$, and $\left.\mathrm{Cu}_{3}\left[\mathrm{Fe}(\mathrm{CN})_{6}\right]_{2}\right)$, which do not display any spin transition. These compounds are structurally very close to bistable systems, such as $\mathrm{CsCo}\left[\mathrm{Fe}(\mathrm{CN})_{6}\right]$ [30], which exhibit bistability and can thus be used as structural models but without the complications which may arise due to the presence of multiple (HS and LS) phases in various proportions in the different particles. We synthesised nanoparticles with different sizes [Fig. 3(a), inset] with a similar crystal structure and environment (for more information see the Supplemental Material [28]). The size dependence of the stiffness variation of these "structural models" provides thus information on the size dependence of $\Gamma$ in bistable analogues. For a quantitative comparison of the stiffness of the particles, we have determined their Debye temperature from the temperature dependence of the total area of their ${ }^{57} \mathrm{Fe}$ Mössbauer spectra (see Supplemental Material [28] for further information). For large particles no significant difference between the Debye temperature of the bulk and the nanoparticles can be detected, but for very small particles (2-3 nm diameter) $\Theta_{D}$ increases for the three compounds which signifies a hardening of the material [Fig. 3(a)]. For the $\mathrm{Fe}_{4}\left[\mathrm{Fe}(\mathrm{CN})_{6}\right]$ system, the slight increase of the Debye temperature at very low size may be attributed to the difference of stiffness between the bulk and the surface. In the $\mathrm{Ni}_{3}\left[\mathrm{Fe}(\mathrm{CN})_{6}\right]_{2}$ compounds, the increase of $\Theta_{\mathrm{D}}$ is more spectacular $(\approx 40 \mathrm{~K})$. A possible explanation may be the occurrence of a reduction from $\mathrm{Fe}^{\mathrm{III}}$ to $\mathrm{Fe}^{\mathrm{II}}$ at the surface of the particles as inferred from the Mössbauer (see Supplemental Material [28]), Raman, and IR spectroscopy analysis. A similar conclusion was reached with the $\mathrm{Cu}_{3}\left[\mathrm{Fe}(\mathrm{CN})_{6}\right]_{2}$ analogue (see Supplemental Material [28]).

While the microscopic origin of the size-dependent variation of stiffness may be different from sample to sample (surface relaxation, surface redox process, etc.) the overall conclusion is the same: The surface atoms differ from the bulk, and their weight on the global elastic properties of the particle increases with decreasing size. 

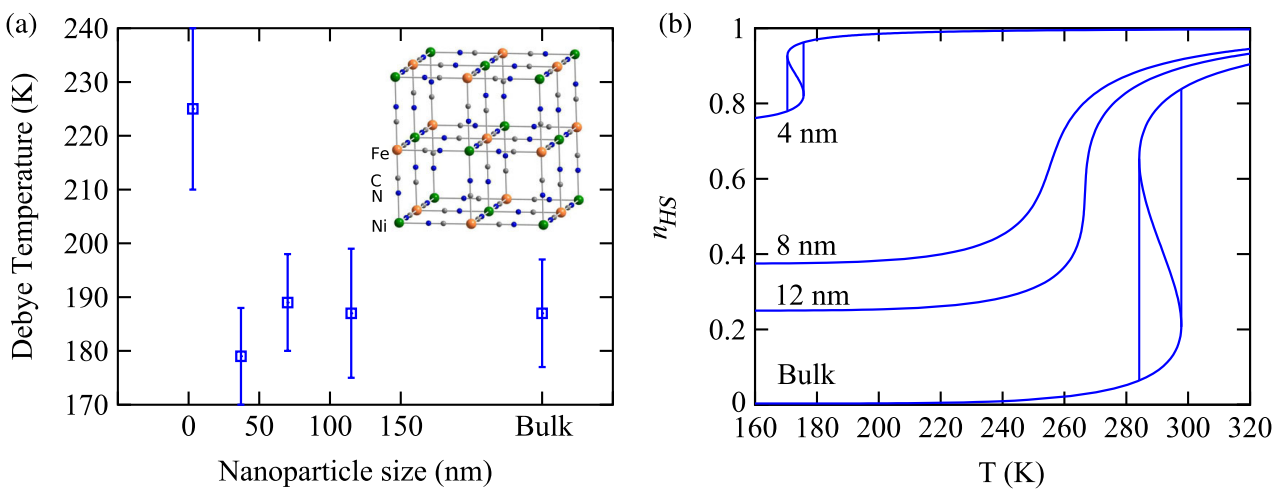

FIG. 3 (color online). (a) Evolution of the Debye temperature for $\mathrm{Ni}_{3}\left[\mathrm{Fe}(\mathrm{CN})_{6}\right]$ Prussian blue analogue nanoparticles as a function of their average size $\left(2 \mathrm{~nm}, 35 \mathrm{~nm}, 70 \mathrm{~nm}, 115 \mathrm{~nm}\right.$, bulk) determined from ${ }^{57} \mathrm{Fe}$ Mössbauer spectroscopy data. Inset: The cubic Prussian blue analogue structure (the presence of alkaline ions and vacancies have been omitted for clarity). (b) Thermal evolution of the total HS fraction for different nanoparticle sizes calculated with the thermodynamic model. The $4 \mathrm{~nm}$ nanoparticle has a $K_{\mathrm{LS}}=40 \mathrm{GPa}$ and a $K_{\mathrm{HS}}=38 \mathrm{GPa}$; the other sizes have a $K_{\mathrm{LS}}=24 \mathrm{GPa}$ and a $K_{\mathrm{HS}}=18 \mathrm{GPa}$.

The influence of this increased rigidity on the spin transition behavior may be important. Figure 3(b) shows a series of size-dependent spin transition curves by taking into account the enhanced stiffness of the small particles. The increase of $\Gamma$ with the diminution of size leads to stronger interactions between the molecules, resulting in the reopening of the hysteresis loop. This finding may well explain the unexpected large hysteresis observed experimentally in ca. $3 \mathrm{~nm}$ SCO particles [18,20,23]. This result opens up new questions about the notion of cooperativity and intermolecular interaction strength when the system reaches a nanometric size. Indeed, cooperativity corresponds to collective effects of entities connected between each other, while the strength of intermolecular interactions depends on the nature of the connections between molecules. The decrease of the size leads to a decrease of the number of connections. If the strength of intermolecular interactions is constant when the size decreases, the system loses the bistability and hysteretic behavior. However, if the intermolecular interactions become stronger at low size, a competition may exist between the two effects and the hysteresis can reappear at very low sizes.

In summary, our results highlight that the cooperativity in nanoscale spin transition materials depends on various surface phenomena. A simple "effective" surface energy term can take into account in a phenomenologic way the differences between the bulk material and particles with high surface to volume ratio. The difference of $\sigma_{\mathrm{HS}}$ and $\sigma_{\mathrm{LS}}$ surface energy leads to a residual fraction at the surface, a shift of the transition temperature, and a loss of cooperativity. In addition, experimental results brought out an enhancement of the elastic modulus of coordination nanoparticles. If this observation is taken into account in the model, we can conclude that it is even possible to recover the hysteresis at very low sizes $(<$ ca. 5-10 nm), due to an enhancement of the elasticity. We shall note that this model was used to investigate spin transition materials, but the fundamental conclusions are readily extendable for a broad family of phase transition materials displaying strong spin-lattice or electron-lattice couplings [31] as well as polymorphism [32]. The common feature of these materials is the central role of the elasticity and a significant volume change upon the phase transition.

It would be very interesting in a further work to develop the surface energy in different terms, i.e., chemical, mechanical, and electrostatic. In addition, specific kinetic aspects such as the heterogeneous nucleation mechanism should also be taken into account [33]. Further experimental work along the predictions of our model will also be necessary and should lead to the development of a new class of phase change materials based on "interface engineering" and/or adjustable mechanical properties.

The authors thank Talal Mallah and Laure Catala for pertinent discussions and financial support from the projects Cross-Nanomat (ANR-10-BLAN-716-1) and Train $^{2}$ SUDOE.

*azzedine.bousseksou@1cc-toulouse.fr

[1] C. Bréchignac, P. Houdy, and M. Lahmani, Nanomaterials and Nanochemistry (Springer, Berlin, 2007).

[2] P. R. Couchman and W. A. Jesser, Nature (London) 269, 481 (1977).

[3] A. N. Goldstein, C. M. Echer, and A. P. Alivisatos, Science 256, 1425 (1992).

[4] V. Swamy, A. Kuznetsov, L. S. Dubrovinsky, R. A. Caruso, D. G. Shchukin, and B.C. Muddle, Phys. Rev. B 71, 184302 (2005).

[5] B. Gilbert, H. Zhang, B. Chen, M. Kunz, F. Huang, and J. F. Banfield, Phys. Rev. B 74, 115405 (2006).

[6] R. Lopez, T. E. Haynes, L. A. Boatner, L. C. Feldman, and R. F. Haglund, Phys. Rev. B 65, 224113 (2002). 
[7] P. Gütlich, A. Hauser, and H. Spiering, Angew. Chem., Int. Ed. Engl. 33, 2024 (1994).

[8] A. Bousseksou, G. Molnar, L. Salmon, and W. Nicolazzi, Chem. Soc. Rev. 40, 3313 (2011).

[9] H. J. Shepherd, G. Molnár, W. Nicolazzi, L. Salmon, and A. Bousseksou, Eur. J. Inorg. Chem. 2013, 653 (2013).

[10] T. Kawamoto and S. Abe, Chem. Commun. (Cambridge) 31, 3933 (2005).

[11] A. Muraoka, K. Boukheddaden, J. Linares, and F. Varret, Phys. Rev. B 84, 054119 (2011).

[12] L. Stoleriu, P. Chakraborty, A. Hauser, A. Stancu, and C. Enachescu, Phys. Rev. B 84, 134102 (2011).

[13] A. Atitoaie, R. Tanasa, and C. Enachescu, J. Magn. Magn. Mater. 324, 1596 (2012).

[14] I. Boldog, A. Gaspar, V. Martínez, P. Pardo-Ibañez, V. Ksenofontov, A. Bhattacharjee, P. Gütlich, and J. Real, Angew. Chem., Int. Ed. 47, 6433 (2008).

[15] F. Volatron, L. Catala, E. Rivière, A. Gloter, O. Stéphan, and T. Mallah, Inorg. Chem. 47, 6584 (2008).

[16] T. Forestier, A. Kaiba, S. Pechev, D. Denux, P. Guionneau, C. Etrillard, N. Daro, E. Freysz, and J.-F. Létard, Chem. Eur. J. 15, 6122 (2009).

[17] A. Rotaru, F. Varret, A. Gindulescu, J. Linares, A. Stancu, J.-F. Létard, T. Forestier, and C. Etrillard, Eur. Phys. J. B 84, 439 (2011).

[18] J. Larionova, L. Salmon, Y. Guari, A. Tokarev, K. Molvinger, G. Molnár, and A. Bousseksou, Angew. Chem., Int. Ed. 47, 8236 (2008).

[19] J. R. Galán-Mascarós, E. Coronado, A. Forment-Aliaga, M. Monrabal-Capilla, E. Pinilla-Cienfuegos, and M. Ceolin, Inorg. Chem. 49, 5706 (2010).

[20] A. Tokarev, L. Salmon, Y. Guari, G. Molnár, and A. Bousseksou, New J. Chem. 35, 2081 (2011).
[21] I. A. Gural'skiy, C. M. Quintero, G. Molnár, I. O. Fritsky, L. Salmon, and A. Bousseksou, Chem. Eur. J. 18, 9946 (2012).

[22] Y. Raza, F. Volatron, S. Moldovan, O. Ersen, V. Huc, C. Martini, F. Brisset, A. Gloter, O. Stéphan, A. Bousseksou, L. Catala, and T. Mallah, Chem. Commun. (Cambridge) 47, 11501 (2011).

[23] P. Durand, S. Pillet, E.-E. Bendeif, C. Carteret, M. Bouazaoui, H. El Hamzaoui, B. Capoen, L. Salmon, S. Hebert, J. Ghanbaja, L. Aranda, and D. Schaniel, J. Mater. Chem. C 1, 1933 (2013).

[24] Q. Jiang, L. H. Liang, and D. S. Zhao, J. Phys. Chem. B 105, 6275 (2001).

[25] C. P. Slichter and H. G. Drickamer, J. Chem. Phys. 56, 2142 (1972).

[26] H. Spiering, K. Boukheddaden, J. Linares, and F. Varret, Phys. Rev. B 70, 184106 (2004).

[27] N. N. Greenwood and T. C. Gibb, Mössbauer Spectroscopy (Chapman and Hall, London, 1971).

[28] See Supplemental Material at http://link.aps.org/ supplemental/10.1103/PhysRevLett.110.235701 for details of the theoretical model as well as sample synthesis and characterisation.

[29] Q.F. Gu, G. Krauss, F. Gramm, A. Cervellino, and W. Steurer, Phys. Rev. Lett. 100, 045502 (2008).

[30] V. Escax, A. Bleuzen, C. Cartier dit Moulin, F. Villain, A. Goujon, F. Varret, and M. Verdaguer, J. Am. Chem. Soc. 123, 12536 (2001).

[31] E. U. Donev, R. Lopez, L. C. Feldman, and R. F. Haglund, Nano Lett. 9, 702 (2009).

[32] S. H. Tolbert and A. P. Alivisatos, Science 265, 373 (1994).

[33] S. Bedoui, M. Lopes, W. Nicolazzi, S. Bonnet, S. Zheng, G. Molnár, and A. Bousseksou, Phys. Rev. Lett. 109, 135702 (2012). 